

\title{
MIDIENDO LA POBREZA ENERGÉTICA. UNA REVISIÓN DE INDICADORES
}

\author{
MEASURING FUEL POVERTY. A REVIEW OF INDICATORS
}

\author{
RAÚL CASTAÑO-ROSA \\ Doctor Arquitecto \\ Investigador posdoctoral Grupo de Tecnologías Apropiadas para \\ el Desarrollo Sostenible, Universidad Carlos III de Madrid \\ Madrid, España \\ https://orcid.org/0000-0002-4459-0220 \\ raulcastano90@gmail.com
}

\author{
JAIME SOLÍS-GUZMÁN \\ Doctor ingeniero Industrial \\ Docente Titular Departamento de Construcciones \\ Arquitectónicas II - Escuela Técnica Superior de Ingeniería de \\ Edificación, Universidad de Sevilla, Sevilla, España \\ https://orcid.org/0000-0001-5535-8112 \\ jaimesolis@us.es
}

\author{
MADELYN MARRERO \\ Doctora Ingeniera Industrial \\ Arquitectónicas II - Escuela Técnica Superior de Ingeniería de \\ Edificación, Universidad de Sevilla, Sevilla, España \\ https://orcid.org/0000-0002-9509-4374 \\ madelyn@us.es
}

\section{RESUMEN}

Identificar aquellos hogares en una situación de vulnerabilidad a la pobreza energética es el primer paso para abordar una problemática social a nivel mundial asociada a la falta de servicios energéticos mínimos, conocido por los términos anglosajones-Fuel Poverty y Energy Poverty, FP y EP, respectivamente. El concepto FP, definido en el Reino Unido como "la incapacidad para obtener un adecuado confort térmico debido a la ineficiencia de la vivienda", mientras que el concepto EP refleja

la imposibilidad de tener acceso a un servicio energético mínimo en países en desarrollo. La falta de un consenso a la hora de definir una ruta clara ha originado que algunos países no la reconozcan como un problema social. La investigación se basa en la revisión de ambos conceptos, a través del análisis conceptual de los términos FP y EP, revisión de indicadores utilizados, estudio de la capacidad de los indicadores para identificar y proponer soluciones a la problemática. Todo ello en relación a los objetivos incluidos: infraestructuras disponibles, eficiencia energética, pobreza social y económica, bienestar y salud social. El resultado es la revisión desde una perspectiva técnica en el sector residencial que ayude a desarrollar soluciones que cubran las carencias encontradas.

\section{Palabras clave}

eficiencia energética, exclusión social, higiene, pobreza

\section{ABSTRACT}

Identifying those households in an energy poverty vulnerability situation is the first step towards addressing a global social problem associated with the lack of minimum energy services, known as Fuel Poverty and Energy Poverty, FP and EP, respectively. The FP concept is defined in the United Kingdom as "the inability to obtain adequate thermal comfort due to the inefficiency of the house", while the EP concept reflects the impossibility in developing countries of having access to a minimal energy service. The lack of consensus when defining a clear path has meant that some countries have not recognized it as a social problem. The research is based on the review of both concepts, through the conceptual

analysis of the terms, FP and EP, a review of indicators used, and the study of the capacity of the indicators to identify and propose solutions to the problem. All this regarding the objectives included: available infrastructures, energy efficiency, social and economic poverty, well-being and social health. The result is a review from a technical perspective in the residential sector, that helps develop solutions that cover the deficiencies found.

Keywords

energy efficiency, social exclusion, hygiene, poverty 


\section{INTRODUCCIÓN}

La pobreza energética (PE), concebida comúnmente como la incapacidad de un hogar en satisfacer una cantidad mínima de servicios de energía para sus necesidades básicas (Castaño-Rosa, Solís-Guzmán, Rubio-Bellido y Marrero, 2019) como por ejemplo, mantener la vivienda en unas condiciones de climatización adecuadas para la salud (Sokołowski, Lewandowski, Kiełczewska y Bouzarovski, 2020), ha suscitado el interés de los gobiernos y partidos políticos, logrando así una mayor repercusión pública. Varias son las definiciones e indicadores desarrollados por los países para analizar la situación de los hogares más vulnerables, entre los que destacan los de Reino Unido, Irlanda, Francia, Eslovaquia, Italia o Austria (Thomson, Snell y Liddell, 2016). Se estima que casi el $20 \%$ de la población total de la Unión Europea estaría incluida en dicha categoría ((EnAct), n.d.). Sin embargo, no existe un concepto oficial común en toda Europa que permita analizar la situación de pobreza energética en los estados miembros y que facilite la comparativa de los resultados obtenidos, con el fin de identificar medidas efectivas para una eventual erradicación.

La Comisión Europea (CE) utiliza tres criterios básicos para evaluar una situación de PE: la incapacidad para mantener las viviendas acondicionadas de manera adecuada, el retraso en el pago de las facturas de servicios públicos y habitar viviendas insalubres (goteras en techos, paredes o suelos, aparición de moho y podredumbre), datos recogidos mediante el Observatorio Europea de la Pobreza Energética (European Commission, 2018). El concepto PE no es únicamente la dificultad para mantener una vivienda a una temperatura adecuada durante las diferentes épocas del año o para hacer frente al pago asociado a un consumo energético o para costear un elevado precio de la energía consumida, sino un concepto multidimensional que ha ido evolucionando, definiéndose actualmente como aquella situación que puede privar a los hogares no sólo de calefacción o refrigeración, sino también de agua caliente, luz y otras necesidades domésticas esenciales (Bouzarovski y Petrova, 2015).

Actualmente, FP y EP son los dos principales conceptos utilizados para identificar una de las mayores problemáticas sociales asociada a la falta de servicios energéticos mínimos en los hogares para cubrir las necesidades básicas, tales como alimentación, higiene personal, zona de confort, seguridad en el hogar, etc. En definitiva, unos servicios energéticos mínimos que garanticen la salud y bienestar social, independientemente de la zona en la que se localice la vivienda, su situación social y económica, estado de salud o país de origen (extranjeros). El objetivo principal del presente trabajo es realizar una revisión de los trabajos y proyectos internacionales más utilizados para identificar de forma efectiva aquellos hogares en riesgo o que se encuentran ya en una situación de FP o EP.

\section{METODOLOGÍA}

Para analizar el concepto PE es necesario desarrollar el estado de la cuestión desde una perspectiva internacional, comenzando por 1) el análisis de los términos anglosajones: Energy Poverty (EP) y Fuel Poverty; siguiendo con 2) la revisión de indicadores utilizados para analizar una situación de pobreza energética; y finalizando con 3) la identificación de la capacidad de los indicadores para solucionar la problemática en relación a los objetivos propuestos: infraestructuras disponibles, eficiencia energética, pobreza social y económica, bienestar y salud social, etc. Dichos objetivos son establecidos en base a los factores de vulnerabilidad energética definidos por Bouzarovski, Petrova y Tirado-Herrero (2014). El resultado es una revisión de los conceptos EP y FP desde una perspectiva técnica en relación al sector residencial, la cual permitirá desarrollar soluciones que cubran las carencias encontradas.

En el examen de indicadores utilizados para analizar una situación de pobreza energética, estos se agrupan en dos categorías: aquellos basados en gastos e ingresos del hogar y aquellos basados en encuestas de percepciones y declaraciones de los hogares. Además, existen indicadores y metodologías que describen aquellos consumidores más vulnerables, como son los análisis econométricos, de la sobreocupación de viviendas comunes, de confort térmico y los fundamentados en la calificación de eficiencia energética de las viviendas. Los indicadores recogidos en la Tabla 1 serán discutidos en las siguientes secciones del artículo.

\section{REVISIÓN DE INDICADORES RELACIONADOS}

Para analizar el PE, es necesario desarrollar el estado de la cuestión desde una perspectiva internacional y, especialmente, el análisis de los términos anglosajones: Fuel Poverty (FP) y Energy Poverty (EP). Este artículo analiza y revisa los conceptos EP y FP, así como los indicadores disponibles más utilizados, desde su capacidad para identificar hogares en riesgo de PE a partir de una perspectiva técnica relacionada con el sector residencial.

El concepto FP fue introducido por Isherwood y Hancock en 1979 tras el forzado aumento del precio de la energía, debido a la crisis del petróleo (1973-1974). Sin embargo, no es hasta el año 1991 cuando Brenda Boardman (2010) define por primera vez el concepto de FP, referido al Reino Unido, como: "la incapacidad para obtener un adecuado confort térmico debido a la ineficiencia de la vivienda", por lo que estableció la posibilidad de que aquellos que son pobres energéticamente no tienen por qué serlos económicamente.

Actualmente, existen diferentes definiciones oficiales de FP desarrolladas en países como Reino Unido, Francia, Irlanda, Eslovaquia (Thomson et al., 2016), así como 


\begin{tabular}{|c|c|}
\hline \multirow{6}{*}{$\begin{array}{l}\text { Basados en } \\
\text { gastos e } \\
\text { ingresos del } \\
\text { hogar }\end{array}$} & Gasto del consumo energético superior al 10\% del ingreso familiar (10\%) (Boardman, 2012) \\
\hline & Gasto del consumo energético superior al doble de la mediana nacional (2M) (Schuessler, 2014) \\
\hline & $\begin{array}{c}\text { Ingreso familiar inferior al Mínimo Ingreso Standard (MIS) (Moore, 2012)and examines the consequent policy } \\
\text { implications. It explores the definition of vulnerable households and the importance of severity and questions } \\
\text { whether the UK fuel poverty strategy is targeted at households least able to afford their fuel costs (as the name } \\
\text { implies }\end{array}$ \\
\hline & $\begin{array}{c}\text { Ingreso familiar inferior al umbral de pobreza monetaria y gasto del consumo energético superior al umbral } \\
\text { establecido (LIHC) (Hills, 2012) }\end{array}$ \\
\hline & $\begin{array}{l}\text { Ingreso familiar después del coste de combustible inferior al umbral establecido al cual se excluye el coste de } \\
\text { combustible medio de la zona analizada (AFCP) (Romero, Linares, López Otero, Labandeira y Pérez Alonso, 2015) }\end{array}$ \\
\hline & Gasto absoluto del consumo energético inferior al umbral establecido (HEP) (Rademaekers et al., 2016) \\
\hline \multirow{4}{*}{$\begin{array}{l}\text { Basados en } \\
\text { encuestas de } \\
\text { percepciones y } \\
\text { declaraciones } \\
\text { de los hogares }\end{array}$} & $\begin{array}{l}\text { Posibilidad de un hogar para mantener una temperatura adecuada durante la estación fría (European Comission, } \\
\text { 2014) }\end{array}$ \\
\hline & Aparecen retrasos en el pago de los recibos energéticos (European Comission, 2014) \\
\hline & $\begin{array}{l}\text { Aparecen deficiencias en la vivienda, como son goteras, humedades en paredes, suelos, techos o cimientos, o } \\
\text { podredumbre en suelos, marcos de ventanas o puertas (European Comission, 2014) }\end{array}$ \\
\hline & $\begin{array}{c}\text { Capacidad de un hogar para mantener una temperatura fresca durante los meses de verano (Spanish National } \\
\text { Statistics Institute, 2014) }\end{array}$ \\
\hline $\begin{array}{l}\text { Basados } \\
\text { en análisis } \\
\text { econométricos }\end{array}$ & $\begin{array}{l}\text { Influencia de determinados condicionantes demográficos, socioeconómicos y físicos en padecer una situación de } \\
\text { PE (Legendre y Ricci, 2014) }\end{array}$ \\
\hline $\begin{array}{l}\text { Basados en el } \\
\text { confort térmico }\end{array}$ & $\begin{array}{l}\text { Porcentaje de horas en el que las estancias se encuentran en una situación de confort térmico (Sánchez-Guevara, } \\
\qquad \text { Neila Gonzalez y Hernández Aja, 2014) }\end{array}$ \\
\hline $\begin{array}{l}\text { Basados en } \\
\text { la eficiencia } \\
\text { energética de } \\
\text { la vivienda }\end{array}$ & $\begin{array}{c}\text { Influencia de la calidad de la vivienda (consumo energético) con una situación de PE. Mala calidad de la } \\
\text { vivienda ocasiona un mayor consumo energético y, a su vez, una situación de PE (Fabbri, 2015)decision-makers, } \\
\text { technicians, researchers, etc. In Italy, a strategy to solve fuel poverty involves action in order to reduce energy } \\
\text { prices, the AEEG (Italian regulatory authority for electricity gas and water }\end{array}$ \\
\hline \multirow[t]{3}{*}{$\begin{array}{l}\text { Basados } \\
\text { en criterios } \\
\text { combinados }\end{array}$} & $\begin{array}{l}\text { Índice de Hogares Vulnerables. Permite evaluar un hogar, sea o no identificado en una situación de pobreza } \\
\text { energética, identificando qué variable requiere una mayor atención: económica, energética o de confort } \\
\text { térmico. Hace posible incluir la viabilidad económica y técnica de una rehabilitación energética (Castaño-Rosa, } \\
\text { Solís-Guzmán y Marrero, 2018)and assesses the home vulnerability situation regardless of whether or not it is in } \\
\text { fuel poverty by using three dimensions: monetary cost, energy and thermal comfort. The monetary dimension } \\
\text { analyses vulnerability in relation to the available net income to face everyday life. The energy variable assesses } \\
\text { the vulnerability related to the constructive characteristics of the dwelling. Finally, the introduction of the thermal- } \\
\text { comfort variable enables the evaluation of the vulnerability related to the inner temperature of the dwelling } \\
\text { and its perception by occupants. The combination of the different resulting values in each dimension and its } \\
\text { relationship to the quality of life of occupants establishes a hierarchy of vulnerable levels. As a result, a multi- } \\
\text { dimensional index is defined which relates technical aspects (characteristics of the dwelling }\end{array}$ \\
\hline & $\begin{array}{l}\text { Fuel Poverty Potential Risk Index. Permite evaluar el riesgo de un hogar a padecer PE en función del lugar donde } \\
\text { se localice su vivienda en el contexto de Chile, utilizando el modelo de confort adaptativo (Pérez-Fargallo, Rubio- } \\
\text { Bellido, Pulido-Arcas y Trebilcock, 2017) }\end{array}$ \\
\hline & $\begin{array}{l}\text { Energy Poverty Vulnerability Index. De aplicación en Portugal, proporciona un análisis espacial de la PE a partir } \\
\text { de combinar varios indicadores: socioeconómico, climático, energético (Gouveia, Palma y Simoes, 2019) }\end{array}$ \\
\hline
\end{tabular}


diversidad de indicadores para su análisis, ninguno reconocido de manera oficial por la CE. Sin embargo, en búsqueda de mitigar esta problemática, en la estrategia Energy Roadmap 2050 (European Commission, n.d.) implementada por la Comisión, se define al consumidor energético vulnerable como aquella familia abastecida por electricidad y formada por personas cuya edad, estado de salud y bajos ingresos presentan riesgo de exclusión social, así como del riesgo de corte del suministro, además de beneficiarse de medidas de protección social para conseguir el suministro eléctrico mínimo requerido (Peneva, 2016).

El concepto de EP ha adquirido relevancia gracias a diversas investigaciones (Bouzarovski y Petrova, 2015; Shonali Pachauri, 2004). Este se asocia a la falta de un suministro de energía, debido a problemas relacionados con las infraestructuras que la distribuyen. El uso del concepto EP ha facilitado identificar las zonas con escasas infraestructuras, antiguas y en un estado de conservación ineficiente, como son los cascos históricos, las zonas rurales y/o las zonas de exclusión social, que, unido a su escasa actividad económica, continua despoblación y pérdida del atractivo inversor, han motivado un abandono continuado, provocando que sus residentes tengan una peor calidad de vida.

Buen ejemplo de EP es el estudio llevado a cabo en Hungría (Tirado Herrero y Ürge-Vorsatz, 2012)a, donde los edificios analizados mostraban un excesivo gasto en el consumo energético, dificultad para realizar un cambio de suministrador o tipo de combustible debido a las restricciones técnicas e institucionales; o la imposibilidad de reducir el gasto en calefacción a través de actuaciones individuales de eficiencia energética. Este tipo de situaciones origina retrasos e imposibilidad en el pago de las facturas energéticas, desconexión del suministro por parte de la compañía suministradora o reducción del consumo de otras necesidades y servicios básicos, y está asociado principalmente a países pobres, ubicados sobre todo en la zona central y sur de América, África y Asia (Bazilian, Sagar, Detchon y Yumkella, 2010; Birol, 2007).

Más allá de las nociones de FP y EP, la realidad es que ambas definen una situación en la que un hogar no puede satisfacer sus necesidades energéticas básicas (tales como calefacción, refrigeración, iluminación o cocina) (Gatto y Busato, 2020), ya sea por una cuestión material o social. De ahí la actual tendencia de identificar, más que una situación de EP O FP, al consumidor vulnerable, el cual convoca los conceptos "resiliencia" -capacidad de adaptación frente a un estado o situación adversa- (Bouzarovski et al., 2014; O'Brien y Hope, 2010; Welsh, 2014) y "precariedad" -carencia de los medios o recursos suficientes- (Paugan, 1995). A continuación, se realiza una revisión de los indicadores de PE más utilizados y reconocidos internacionalmente
(ASSIST 2GETHER, 2018; Herrero, 2017; Rademaekers et al., 2016; Meszerics, 2016; Thomson et al., 2016), usando como base el Observatorio Europeo de la Pobreza Energética (European Commission, 2018), el Observatorio de Pobreza Energética en México ("Observatorio de Pobreza Energética en México," n.d.), y la Red de Pobreza Energética de Chile ("Red de Pobreza Energética (RedPE), Universidad de Chile", 2017). El análisis de debilidades y amenazas de los indicadores se realiza a partir de los factores de vulnerabilidad energética definidos por Bouzarovski et al. (2014).

\section{INDICADOR DEL 10\%}

Este indicador define que un hogar está en pobreza energética si ha de dedicar más del $10 \%$ de sus ingresos a pagar unos servicios energéticos adecuados (tales como calefacción, refrigeración, iluminación o cocina) (Boardman, 2010). Definido por Boardman, es un indicador simple, fácil de comunicar y relativamente versátil, lo que permite establecer una clara meta política. Las críticas son fundamentalmente debidas, por un lado, a su excesiva sensibilidad a los precios de la energía, infraestimando la escala del problema cuando los precios son bajos y sobreestimándola cuando son altos, $y$, por otro, al carácter arbitrario en la fijación del umbral en el 10\%, un umbral que se justificó en la coyuntura socioeconómica del Reino Unido a comienzo de los 90. La experiencia de años de aplicación ha mostrado que este umbral del 10\% incluyó un número significativo de hogares que no eran pobres energéticos, como hogares de renta alta con casas ineficientes.

\section{INDICADORES 2M}

Estos incluyen: doble de la mediana de gasto en energía del hogar, doble de la media de gasto de energía del hogar, doble de la mediana de porcentaje de gasto en energía del hogar y doble de la media de porcentaje de gasto en energía del hogar (Schuessler, 2014). Sólo el tercero de los indicadores anteriores tiene su justificación en los trabajos pioneros de Boardman, en los cuales se detectó que la mediana del porcentaje de gasto energético respecto al total de ingresos en los hogares británicos rondaba el 5\% para el año 1988. Tras ello, la evaluación aportada por estos indicadores indica que "Un hogar está en pobreza energética si de sus ingresos ha de dedicar más del doble de la mediana de porcentaje de gasto en energía para pagar unos servicios energéticos adecuados" (Schuessler, 2014 , p. 11). El umbral de PE se establece en relación a la media nacional, posibilitando que sea recalculado cada año. En este sentido, no es una medida estática. Como puntos fuertes del indicador se puede destacar: los hogares con altos ingresos son raramente incluidos como pobres energéticos y tiene en consideración las características específicas del país. 


\section{MÍNIMO INGRESO STANDARD (MIS)}

EI MIS, definido por Moore (2012), considera aquella renta mínima de un hogar que permita a sus integrantes optar a las oportunidades y elecciones que, a su vez, les posibiliten una integración activa en la sociedad. El proyecto "A minimum income standard for Britain" desarrollado por Bradshaw et al. (2008) representa un buen ejemplo de lo que esta metodología pretende. Lo primero a subrayar es que se trata de un proyecto acotado, ya que establece un MIS siempre asociado a un colectivo social concreto y son las personas escogidas como representantes de diversos colectivos sociales las que participarán en todo el proceso deliberativo y en la elaboración de las conclusiones. Definir lo que se entiende por "ingreso mínimo aceptable" (MIS) es la principal limitación. Este proyecto se basa en la Convención de Derechos Humanos de las ONU y los trabajos de un comité de expertos en los EE.UU. que realizaron una revisión de los presupuestos familiares en 1980 y desarrollaron el concepto de Prevailing Family Standard. Para tener en cuenta a colectivos especialmente vulnerables se establecieron una serie de parámetros socioeconómicos del hogar: composición del hogar, situación de empleo, discapacidad, salud, etnia y accesibilidad. De este modo, "un hogar se encontraría en situación de pobreza energética si sus ingresos totales menos sus costes energéticos no superasen el MIS correspondiente a las características de su hogar" (Moore, 2012, p. 21).

\section{LOW INCOME HIGH COST (LIHC)}

A partir de los estudios realizados por Hills (2012), se concibe un hogar como pobre energético si sus ingresos se encuentran por debajo de un umbral de pobreza determinado y cuando sus gastos energéticos son superiores a otro umbral para el gasto energético. Para ello es necesario establecer ambos umbrales: el primero se define en el $60 \%$ de la mediana equivalente de ingresos después de restar los gastos de la vivienda y los gastos energéticos. Para el segundo umbral se usó la mediana equivalente de gasto en energía calculado sobre el total de hogares (ver Figura 1).

El punto fuerte de este indicador se basa en la posibilidad de distinguir entre PE y pobreza general, reflejando claramente que la PE es dependiente de los ingresos del hogar. A su vez, el uso de este indicador deja fuera de una situación de PE aquellos grupos considerados más vulnerables (personas mayores, enfermos crónicos, minusválidos y niños pequeños) (Middlemiss, 2016)the UK government transformed the politics of fuel poverty, with a new definition ('Low income, high costs' or LIHC, ya que el hogar definido como pobre energético está formado por unos bajos ingresos familiares y una vivienda energéticamente ineficiente. Esto provoca que mejorar la eficiencia energética de la vivienda sea considerada la principal medida para reducir la $\mathrm{PE}$, obviando que los hogares con bajos ingresos seguirán en cierta medida teniendo problemas para pagar sus facturas y experimentando problemas de salud por vivir en un confort inadecuado.

\section{AFTER FUEL COST POVERTY (AFCP)}

Basado en el indicador inicial del MIS propuesto por Moore, el desarrollo realizado por Heindl (2015) y las aplicaciones realizadas en España por Romero et al. (2015) y en Reino Unido por Hills (2012), considera que un hogar está en pobreza energética cuando los ingresos del hogar, una vez descontados los gastos de vivienda y energía doméstica, caen por debajo del nivel de ingresos mínimos aceptables (ajustados al tamaño y composición del hogar por medio la escala de equivalencia de la OCDE modificada). En

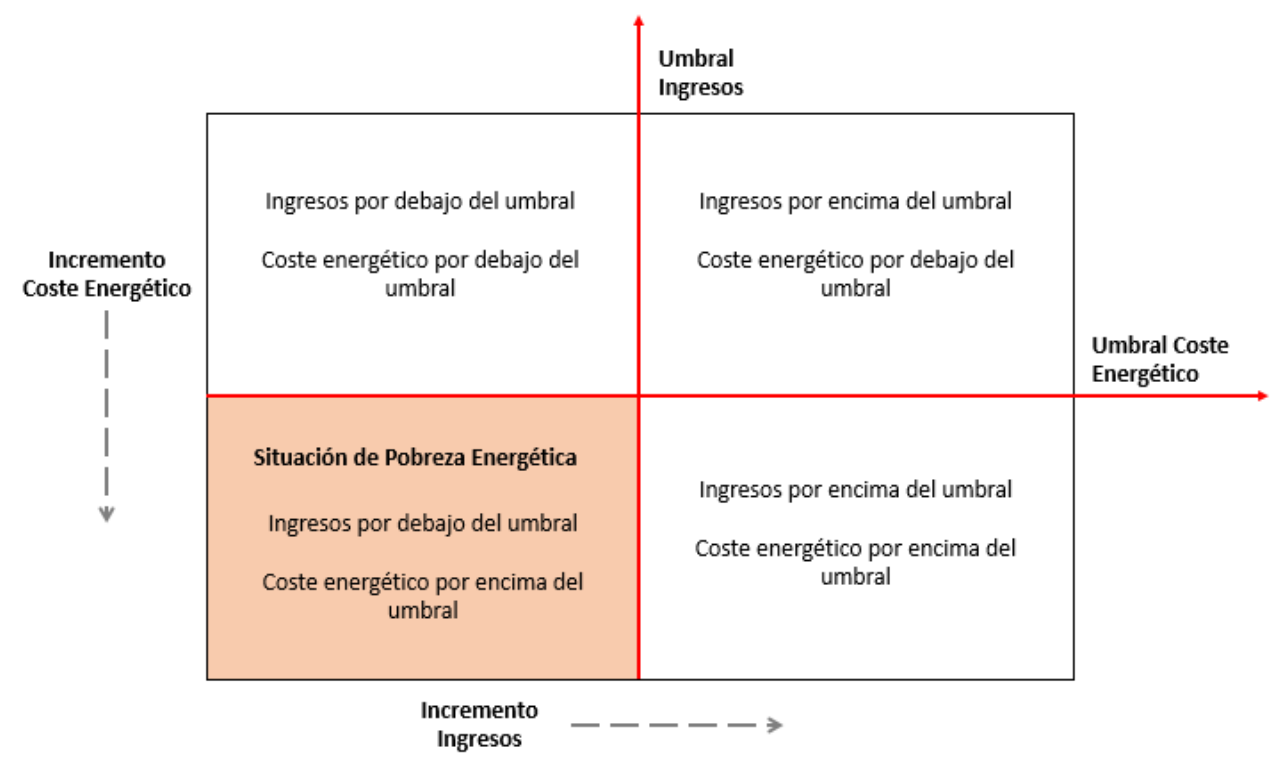


resumen, este enfoque se fundamenta en la existencia de un nivel de ingresos mínimos que garantice el bienestar de una persona, posibilitando que un hogar no sea excluido de la sociedad de la que forma parte (exclusión social) por un factor económico.

\section{HIDDEN ENERGY POVERTY INDICATOR (HEP)}

El HEP identifica aquellos hogares cuyo gasto energético es demasiado bajo, de manera que habrá una situación de PE si el gasto energético total está por debajo de la mediana del gasto energético (Rademaekers et al., 2016). Este indicador permite distinguir a aquellos hogares cuyos ingresos no permiten realizar un consumo energético mínimo debido a tener que priorizar los gastos en alimentación, dando respuesta al conocido efecto "heating or eating". Sin embargo, sólo es válido si se utiliza el gasto monetario absoluto, ya que generalmente los hogares con altos ingresos gastan más en energía en valor absoluto, pero menos como parte proporcional de sus ingresos; de lo contrario, hogares con un gasto muy bajo en relación a sus ingresos, por tener unos ingresos elevados, serían considerados en situación de PE. A su vez, el uso del gasto en valor absoluto permite aportar una evaluación absoluta del consumo de servicios energéticos realizado, identificando aquellos hogares que tienen un gasto por debajo de lo normal, absteniéndose de un nivel básico de consumo. Entre sus limitaciones, hay que señalar que HEP no considera las características de la vivienda, ni su eficiencia energética.

\section{ENCUESTAS DE PERCEPCIONES Y DECLARACIONES DE LOS HOGARES}

Las encuesta de ingresos y condiciones de vida de la Unión Europea (EU-SILC) (European Comission, 2014) tienen como objetivo disponer de una fuente de referencia sobre estadísticas comparativas en el ámbito europeo de los ingresos y la exclusión social. De todos los aspectos de la vida cotidiana de los hogares que analiza esta encuesta, las tres preguntas generalmente utilizadas para el análisis de la PE son relativas a: incapacidad de un hogar para mantener una temperatura adecuada durante la estación fría; retrasos en el pago de recibos, y deficiencias en la vivienda, como son goteras, humedades en paredes, suelos, techos o cimientos, o podredumbre en suelos, marcos de ventanas o puertas.

Las encuestas de percepciones y declaraciones del hogar (ECV) (Spanish National Statistics Institute, 2014; Tirado Herrero, Jiménez Meneses, López Fernández, Martín Gracía y Perero Van Hove, 2014) estudian la situación climatológica cálida padecida en países como España, realizadas por el Instituto Nacional de Estadística, posibilitando una evaluación de las condiciones de vida de las personas en periodos de calor excesivo, al preguntar por la capacidad de un hogar para mantener una temperatura fresca durante los meses de verano. La principal debilidad aquí viene motivada por su carácter subjetivo, susceptible de crear incertidumbre en los resultados.
En resumen, se debe aclarar que estos indicadores no fueron creados para analizar la problemática asociada a la PE, de modo que se hace necesario incluir nuevas variables que permitan establecer una diferencia entre los problemas asociados a la imposibilidad de un consumo energético mínimo y los relacionados a las características de la vivienda o sistemas de calefacción.

\section{ANÁLISIS ECONOMÉTRICOS}

Estos análisis tienen como objetivo explicar una variable a partir de otras, así como de las posibles perturbaciones a las que se pueda ver sometida, analizando su comportamiento. A través de ellos se pretende identificar los colectivos que se encuentran en una situación de mayor vulnerabilidad para padecer una situación de PE, por lo que no identifican una situación de PE en sí. Los estudios de Legendre y Ricci (2014) para Francia y de Miniaci, Scarpa y Valbonesi (2014) para Italia constituyen buenos ejemplos de este tipo de análisis, a través de los cuales se pretende cuantificar la influencia que determinados condicionantes demográficos, socioeconómicos y físicos ejercen en la probabilidad de que un hogar, que a priori no esté en pobreza energética, caiga por debajo del umbral de la misma.

Destacan los modelos desarrollados por Walker, McKenzie, Liddell y Morris (2012) y Walker, Liddell, McKenzie y Morris (2013) que introducen técnicas basadas en los Sistemas de Información Geográfica para elaborar un índice de riesgo de PE, evaluando: tamaño de la familia, consumo eléctrico, nivel de ocupación, precio del combustible utilizado, etc. (Walker, McKenzie, Liddell y Morris, 2014).

\section{CONFORT TÉRMICO}

El confort térmico puede ser entenderse como "esa condición de la mente que expresa satisfacción con el ambiente térmico" (BS/EN 15251:2007, n.d.). La inclusión del consumidor vulnerable ha motivado el estudio del confort térmico de la vivienda principalmente por su estrecha relación a la salud de las personas (Butcher, 2014; Kolokotsa y Santamouris, 2015), además de su capacidad de permitir una reducción del consumo energético de la vivienda (Hatt, Saelzer, Hempel y Gerber, 2012; Martínez y Kelly, 2015; Van Hooff, Blocken, Hensen y Timmermans, 2015), dado que un confort adecuado en la vivienda conlleva, en efecto, el control de dicho consumo energético (Vilches, Barrios Padura y Molina Huelva, 2017).

La evaluación del confort térmico en una vivienda resulta muy complicada (der Perre, Ness, Thoen, Vandenameele y Engels, 2002; Heijs y Stringer, 1988; M. Bluyssen, 2014), fundamentalmente por la gran diversidad de factores que influyen (Bienvenido-Huertas, Rubio-Bellido, Pérez-Fargallo y Pulido-Arcas, 2020). En este sentido, cabe destacar el trabajo de Sánchez-Guevara et al. (2014) en el cual utilizan la evaluación del confort de una vivienda como indicador de hogares más vulnerables. A partir del análisis de los datos obtenidos de la simulación y haciendo uso de modelos 


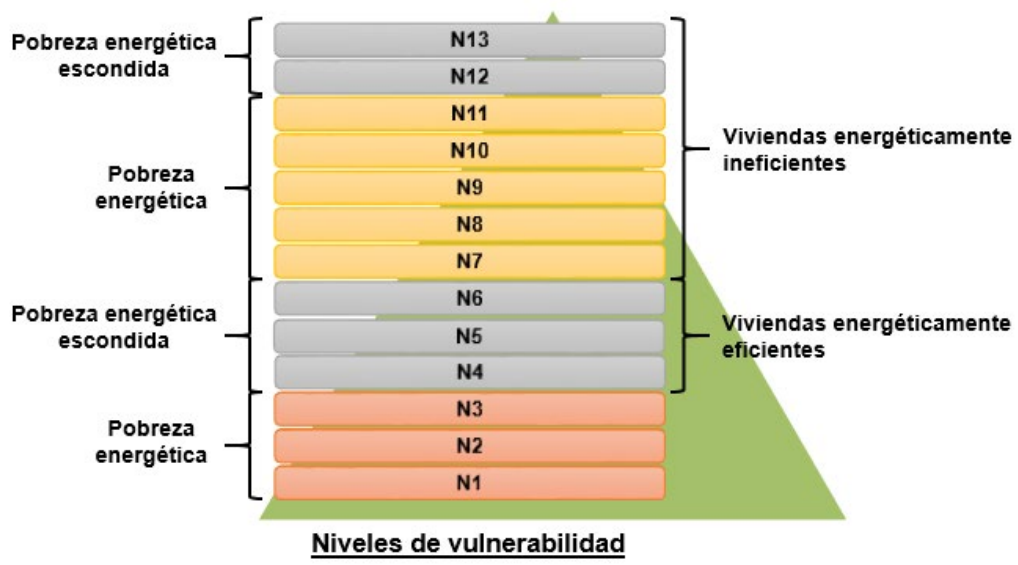

Figura 2. Niveles de vulnerabilidad del Índice de Hogares Vulnerables (IHV). Fuente: Castaño-Rosa (2018, p. 55).

adaptativos para evaluar el confort térmico (ASHRAE (2013) ANSI/ASHRAE Standard 55-2013, 2013)(BS/EN 15251:2007, n.d.), identifican el número de horas que el hogar de estudio se encuentra fuera de la zona de confort establecida. Aquellos hogares con un mayor número de horas fuera de la zona de confort serán considerados como más vulnerables.

\section{EFICIENCIA ENERGÉTICA DEL EDIFICIO}

Debido a la relación establecida entre la eficiencia energética de la vivienda, representada por su consumo energético y la $\mathrm{PE}$, son varios los investigadores que promueven reducir dicha situación a través de una disminución en el consumo energético de la vivienda (Braubach y Ferrand, 2013; Rosenow, Platt y Flanagan, 2013; Boardman, 2012). En esta línea, el caso llevado a cabo en Francia por Florio y Teisser (2015) a través del cual se elabora un certificado de eficiencia energética que, a partir del gasto energético estimado de la vivienda, permite evaluar el stock de viviendas característico en ese país. Otro caso es el realizado en Italia por Fabbri (2015), mediante el cual se propone un indicador de PE basado en el rendimiento energético de la vivienda a partir de tres variables: base de datos de certificación de eficiencia energética, monitorización del consumo energético real y estándar del rendimiento energético de la vivienda en función de su antigüedad. La relación establecida entre los datos de consumo energético de las viviendas y los hogares de bajos ingresos económicos (excesivo consumo energético y bajos ingresos), hace posible señalar los hogares en situación de PE que no tienen suficientes ingresos para llevar a cabo medidas de eficiencia energética, incluso recibiendo incentivos económicos. Recientemente, PorrasSalazar, Contreras-Espinoza, Cartes, Piggot-Navarrete y Pérez-Fargallo (2020), en su último estudio sobre viviendas sociales del centro-sur de Chile, demuestran que un tercio de los entrevistados no pueden mantener una temperatura adecuada en su hogar $y$, como resultado, tienen problemas respiratorios y un mayor gasto médico. Concluye que mejorar la eficiencia energética de dichas viviendas para así conseguir una temperatura adecuada durante mayor periodo de tiempo, permitiría reducir el número de familias con problemas respiratorios, y el gasto médico asociado a ellos.

\section{ÍNDICE DE HOGARES VULNERABLES}

En una combinación de los indicadores descritos en las secciones anteriores se ha propuesto el Índice de Hogares Vulnerables (IHV) (Castaño-Rosa et al., 2018) que permite un análisis de la situación de vulnerabilidad en relación a sus consecuencias y a su intensidad, así como también la posibilidad de evaluar la medida de rehabilitación energética óptima para mejorar la calidad de vida de los hogares. La Figura 2 muestra gráficamente la composición del IHV: 13 niveles (N1: nivel 1 de vulnerabilidad y menos desfavorable; a N13: nivel 13 de vulnerabilidad y más desfavorable), y asimismo la equivalencia a una situación de pobreza energética o pobreza energética escondida. Su última aplicación, en seis comunidades de vecinos localizadas en el área del Casco Norte de Sevilla, las cuales recibieron financiación por parte del Ministerio de Andalucía para llevar a cabo una intervención de mejora de la eficiencia energética, muestra cómo es posible estimar tanto el coste del Servicio Nacional de Salud asociado a una situación de pobreza energética, como el ahorro conseguido tras una intervención de rehabilitación energética (CastañoRosa, Solís-Guzmán y Marrero, 2020). Adicionalmente, el IHV ha sido adaptado y aplicado al contexto británico (Castaño-Rosa, Sherriff, Thomson, Guzmán y Marrero, 2019), sugiriendo que aún hay un importante margen de mejora en la definición de indicadores. El IHV es una nueva herramienta para el análisis e identificación de hogares vulnerables de padecer PE, proporcionando un exhaustivo análisis en la identificación de las diferentes situaciones de vulnerabilidad que un hogar puede padecer (CastañoRosa, Sherriff, Solís-Guzmán y Marrero, 2020; CastañoRosa et al., 2019) . 


\section{FUEL POVERTY POTENTIAL RISK INDEX}

Con el objetivo de evaluar el riesgo de un hogar a padecer PE en función del lugar donde se localice su vivienda en el contexto de Chile, se aporta el Fuel Poverty Potential Risk Index (FPPRI) (Pérez-Fargallo et al., 2017). El uso del modelo de confort adaptativo permite, a partir de la aplicación del FPPRI, considerar la relación entre los ocupantes y la vivienda en la evaluación del riesgo de padecer $\mathrm{PE}$, en especial para climas templados como las regiones del centro de Chile. El uso del confort adaptativo cubre, en parte, el aspecto subjetivo de la interacción de los ocupantes con la vivienda, reduciendo la posibilidad de sobreestimaciones. Sin embargo, la principal limitación del FPPRI es que debe aplicarse en la fase de diseño de la vivienda y no en hogares ya ocupados. En este contexto, cabe destacar el trabajo realizado por Bienvenido-Huertas et al. (2020) en el que se lleva a cabo la aplicación del FPPRI, en las tres ciudades con mayor población de Chile (Santiago, Concepción y Valparaíso), para predecir el riesgo que tendría un hogar a padecer pobreza energética en una vivienda social en función de las características socioeconómicas de los ocupantes y las características técnicas de la vivienda (Bienvenido-Huertas, Pérez-Fargallo, Alvarado-Amador y Rubio-Bellido, 2019). Este trabajo muestra el potencial del FPPRI para reducir el riesgo de un hogar de padecer una situación de PE en un futuro próximo.

\section{ENERGY POVERTY VULNERABILITY INDEX}

La eficiencia energética de la viviendas, la posibilidad de los hogares de implementar medidas y las dificultades en calentar y/o enfriar las viviendas, son los diferentes aspectos analizados por el Energy Poverty Vulnerability Index (EPVI), de aplicación en Portugal (Gouveia et al., 2019). En el caso de estudio definido para su aplicación, donde 3092 distritos fueron analizados, se muestra el potencial del EPVI para identificar las zonas con un mayor riesgo a padecer una situación de PE, permitiendo posteriormente un análisis detallado a nivel local. En definitiva, el EPVI es una herramienta efectiva, de aplicación en Portugal, para la elaboración de políticas nacionales y locales de eficiencia energética. La principal limitación de este índice recae en la disponibilidad de los datos necesarios para su aplicación, lo que imposibilita su aplicación en otros países donde el acceso a la información es más restringido.

\section{DISCUSIÓN}

La calidad de la vivienda es compleja de evaluar y posiblemente la más influyente en la PE de un hogar. Por lo que la eficiencia energética puede ser un instrumento decisivo y efectivo en la reducción de la $\mathrm{PE}$, tal como muestra (Porras-Salazar et al., 2020) influyendo en la calificación energética, la envolvente energética, las instalaciones, el nivel de ventilación, el estado de conservación y la antigüedad del hogar. Dicha evaluación se realiza mediante datos de consumo energético, por lo que todos los indicadores analizados son capaces, de alguna manera, de establecer una relación, ya sea de forma directa o indirecta, entre la eficiencia energética de una vivienda y la PE. Otra metodología que permite relacionar la calidad de la vivienda con la PE consiste en la evaluación del confort térmico, como se muestra en los trabajos desarrollados por Sánchez-Guevara, Neila González y Hernández Aja (2018); Boemi y Papadopoulos (2019) y Porras-Salazar et al. (2020).

Uno de los factores más difusos de evaluar es el impacto social que origina la PE, por ejemplo, la exclusión social. La relación entre la PE y la exclusión social se debe a que las familias reducen las actividades sociales con amigos y conocidos por miedo a ser considerados pobres y/o por no poder proporcionar unas condiciones adecuadas en su vivienda para mantener actividades sociales (Longhurst y Hargreaves, 2019). La inclusión de los ingresos familiares por parte de los indicadores basados en gastos e ingresos del hogar, así como los basados en encuestas de percepciones y declaraciones, permiten relacionar la PE y la situación de pobreza económica y/o social.

Otro aspecto importante lo constituye la salud: está constatado que vivir en una casa con temperaturas inadecuadas origina mayores tasas de admisiones hospitalarias y una mayor incidencia y severidad de síntomas asmáticos (Liddell y Morris, 2010). También se ha identificado que la probabilidad de sufrir depresiones o estrés entre los adolescentes que viven en una casa insuficientemente acondicionada es de más del $25 \%$, mientras que en hogares que no experimentan esta problemática es del 5\% (Howden-Chapman, Viggers, Chapman, O'Sullivan, Telfar Barnard y Lloyd, 2012). Aunque son los ancianos, los niños y las embarazadas, considerados como población vulnerable, quienes tienen una mayor probabilidad para verse afectados por estas enfermedades (Dear y McMichael, 2011). Además de problemas de salud mental, habitar una vivienda con temperaturas inadecuadas en invierno es una causa asociada a tener problemas de salud física como gripe o resfriado, incluso se acredita al empeoramiento de la situación de personas que padecen artritis y reumatismo (Ortiz, Casquero-Modrego y Salom, 2019). A continuación, la Tabla 2 sintetiza las capacidades identificadas durante la revisión de los indicadores.

\section{CONCLUSIÓN}

El objetivo principal de este documento ha sido proporcionar una revisión de los indicadores actuales de pobreza energética más utilizados y reconocidos internacionalmente, siguiendo los criterios definidos en el Observatorio Europeo de la Pobreza Energética, el 
\begin{tabular}{l|l|l|l|l|l|l|l|l|l|l|l|l|}
$10 \%$ & $2 \mathrm{M}$ & MIS & LIHC & AFCP & HEP & Encuestas de & Econométricos & CT & EE & IHV & FPPRI & EPVI
\end{tabular} percepciones

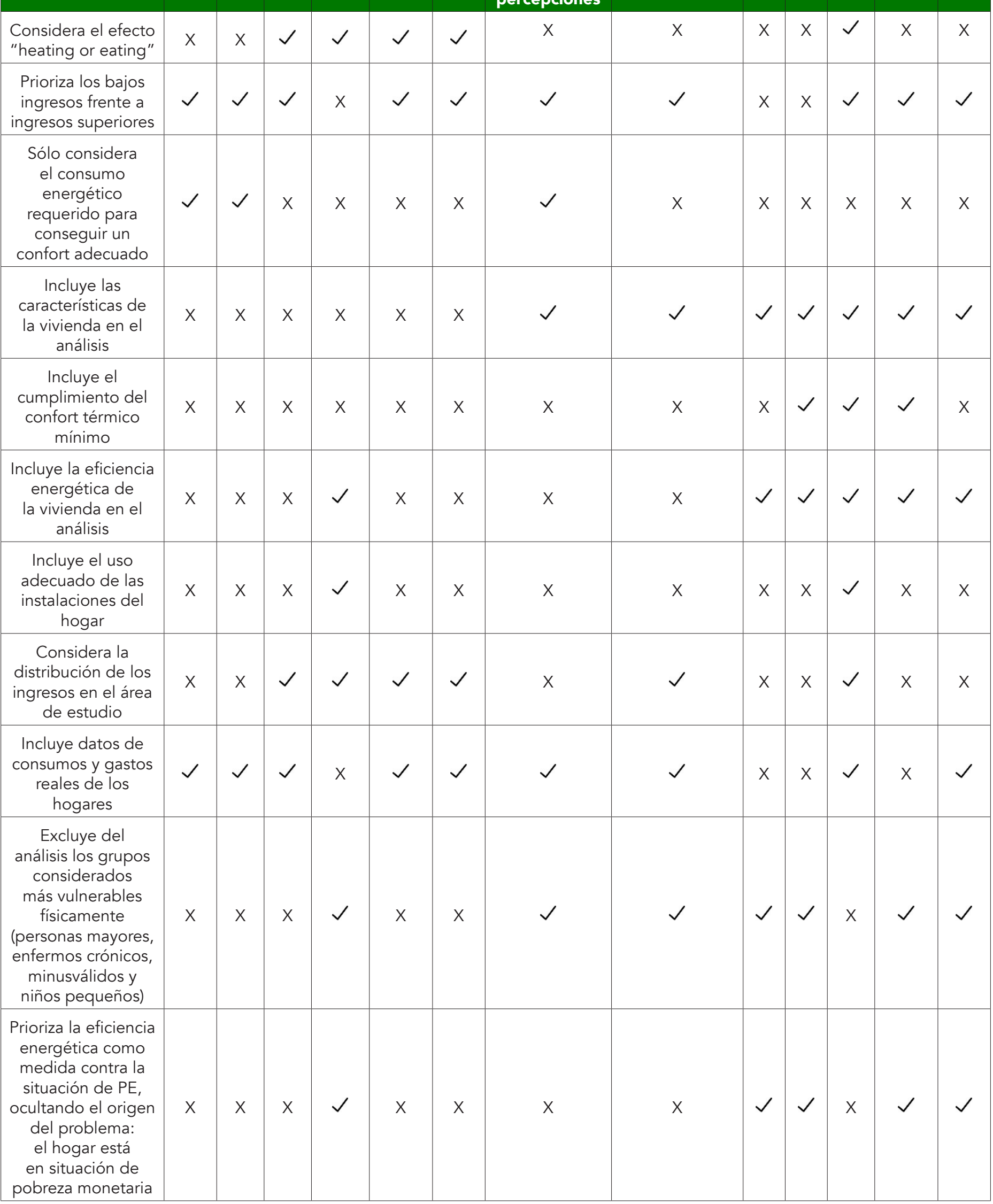

Tabla 2. Análisis crítico de los indicadores analizados.

10\% (indicador del 10\%); $2 \mathrm{M}$ (indicadores 2M); MIS (Mínimo Ingreso Standard); LIHC (Low Income High Cost); AFCP (After Fuel Cost Poverty); HEP (Hidden Energy Poverty); CT (Confort Térmico); EE (Eficiencia Energética); IHV (Índice de Hogares Vulnerables); FPPRI (Fuel Poverty Potential Risk Index); EPVI (Energy Poverty Vulnerability Index). Fuente. Elaboración de los autores. 
Observatorio de Pobreza Energética de México, y la Red de Pobreza Energética de Chile, en cuanto a su capacidad para identificar aquellos hogares en riesgo de padecerla (ver Tabla 2). Con este fin, los factores de vulnerabilidad energética (infraestructuras disponibles, eficiencia energética, pobreza monetaria y social, bienestar y salud), aportados por Bouzarovski et al. (2014), se han empleado para analizar la efectividad de los indicadores actuales de PF, que se pueden agrupar de acuerdo con los indicadores en que se basan: ingresos-gastos; condiciones auto-informadas; análisis econométrico; comodidad térmica; y eficiencia energética.

La principal debilidad de todos estos indicadores radica en la imposibilidad de que un solo indicador considere todos los factores posibles que influyen en la actividad cotidiana de los hogares, como el confort térmico, la salud y el bienestar. En consecuencia, se proporciona un análisis incompleto si se usan de forma aislada, principalmente debido a imprecisiones de exclusión (por lo que los hogares que deberían recibir beneficios no son reconocidos por las estrategias de los gobiernos) e inexactitudes en la inclusión (debido a las cuales los hogares que no están en riesgo de sufrir pobreza energética cumplen con los criterios de elegibilidad $y$, por lo tanto, reciben apoyo). Por lo tanto, es necesario combinar varios indicadores y analizar sus resultados para determinar si se logra un análisis holístico tanto de las características técnicas de la vivienda como de la situación del hogar.

La discusión presentada en este documento expone las debilidades de los indicadores de PE existentes en la identificación de hogares en riesgo y conduce a la definición de un enfoque de indicadores múltiples que reúna tantos factores como sea posible. Además, debido a la complejidad de extrapolar los indicadores definidos a otros países, o zonas climáticas, con diferente contexto social y económico, se argumenta la necesidad de que cada país defina la PE acorde a las circunstancias del contexto para desarrollar políticas concretas y eficaces. Por último, en el caso particular de la Unión Europea, la falta de definiciones e indicadores adecuados en la mayoría de los estados miembros lleva a esta investigación a proporcionar un punto de partida.

\section{AGRADECIMIENTOS}

Esta investigación forma parte del proyecto Nuevo Análisis Integral de la Pobreza Energética en Andalucía (NAIPE). Predicción, evaluación y adaptación al cambio climático de hogares vulnerables desde una perspectiva económica, ambiental y social. Referencia US-1255465. Financiado dentro de la convocatoria de Proyectos I+D+i FEDER Andalucía 2014-2020 por la Consejería de Economía y Conocimiento de la Junta de Andalucía (España).

\section{REFERENCIAS BIBLIOGRÁFICAS}

ASHRAE (2013) ANSI/ASHRAE Standard 55-2013. Thermal Environmental Conditions for Human Occupancy (2013). Atlanta.

ASSIST 2GETHER. (2018). REPORT ON NATIONAL AND EUROPEAN MEASURES ADDRESSING VULNERABLE CONSUMERS AND ENERGY POVERTY. EU. Recuperado de https://www.assist2gether.eu/documenti/risultati/report_on_ national_and_european_measures_addressing_vulnerable_ consumers_and_energy_poverty.pdf

Bazilian, M., Sagar, A., Detchon, R. y Yumkella, K. (2010). More heat and light. Energy Policy 2010, 38(10), 5409-5412. https:// doi.org/10.1016/j.enpol.2010.06.007

Bienvenido-Huertas, D., Pérez-Fargallo, A., Alvarado-Amador, R. y Rubio-Bellido, C. (2019). Influence of climate on the creation of multilayer perceptrons to analyse the risk of fuel poverty. Energy and Buildings, 198, 38-60. https://doi.org/https://doi. org/10.1016/j.enbuild.2019.05.063

Bienvenido-Huertas, D., Rubio-Bellido, C., Pérez-Fargallo, A. y Pulido-Arcas, J. A. (2020). Energy saving potential in current and future world built environments based on the adaptive comfort approach. Journal of Cleaner Production, 249, 119306. https:// doi.org/https://doi.org/10.1016/j.jclepro.2019.119306

Birol, F. (2007). Energy Economics: A Place for Energy Poverty in the Agenda? Energy Journal 2007, 28(3), 1-6.

Boardman, B. (2010). Fixing Fuel Poverty. Challenges and Solutions. London: Earthscan.

Boardman, B. (2012). Achieving zero. Delivering future-friendly buildings. Oxford: Oxford.

Boemi, S. N. y Papadopoulos, A. M. (2019). Energy poverty and energy efficiency improvements: A longitudinal approach of the Hellenic households. Energy and Buildings, 197, 242-250. https:// doi.org/https://doi.org/10.1016/j.enbuild.2019.05.027

Bouzarovski, S., Petrova, S. y Tirado-Herrero, S. (2014). From Fuel Poverty to Energy Vulnerability: The Importance of Services, Needs and Practices. (No. Science Policy Research Unit). Manchester. Retrieved from http://www.sussex.ac.uk/spru/ research/swps

Bouzarovski, S. y Petrova, S. (2015). A global perspective on domestic energy deprivation: Overcoming the energy povertyfuel poverty binary. Energy Research and Social Science, 10(10), 31-40. https://doi.org/10.1016/j.erss.2015.06.007

Bradshaw, J., Middleton, S., Davis, A., Oldfield, N., Smith, N., Cusworth, L. y Williams, J. (2008). A Minimum Income Standard for Britain: What people think. York: Joseph Rowntree Foundation.

Braubach, M. y Ferrand, A. (2013). Energy efficiency, housing, equity and health. International Journal of Public Health 2013, 58(3), 331-332. https://doi.org/10.1007/s00038-012-0441-2

BS/EN 15251:2007. Indoor environmental input parameters for design and assessment of energy performance of buildings addressing indoor air quality, thermal environment, lighting and acoustics. London: BSI, 2007. 
Butcher, J. (2014). Fuel Poverty How To Improve Health and Wellbeing Through Action on Affordable Warmth. London: UK Health Forum 2014.

Castaño-Rosa, R. (2018). Identificación de hogares vulnerables a partir del concepto pobreza energética. Indicador y modelo de evaluación. University of Seville.

Castaño-Rosa, R., Sherriff, G., Solís-Guzmán, J. y Marrero, M. (2020). The validity of the index of vulnerable homes: evidence from consumers vulnerable to energy poverty in the UK. Energy Sources, Part B: Economics, Planning, and Policy, 1-20. https:// doi.org/10.1080/15567249.2020.1717677

Castaño-Rosa, R., Sherriff, G., Thomson, H., Guzmán, J. S. y Marrero, M. (2019). Transferring the index of vulnerable homes: Application at the local-scale in England to assess fuel poverty vulnerability. Energy and Buildings, 203, 109458. https://doi. org/https://doi.org/10.1016/j.enbuild.2019.109458

Castaño-Rosa, R., Solís-Guzmán, J. y Marrero, M. (2018). A novel Index of Vulnerable Homes: Findings from application in Spain. Indoor and Built Environment, 1420326X18764783. https://doi. org/10.1177/1420326X18764783

Castaño-Rosa, R., Solís-Guzmán, J. y Marrero, M. (2020). Energy poverty goes south? Understanding the costs of energy poverty with the index of vulnerable homes in Spain. Energy Research \& Social Science, 60, 101325. https://doi.org/https://doi. org/10.1016/j.erss.2019.101325

Castaño-Rosa, R., Solís-Guzmán, J., Rubio-Bellido, C. y Marrero, M. (2019). Towards a multiple-indicator approach to Energy Poverty in the European Union: a review. Energy and Buildings, 193, 36-48. https://doi.org/https://doi.org/10.1016/j. enbuild.2019.03.039

Dear, K. B. G. y McMichael, A. J. (2011). The health impacts of cold homes and fuel poverty. BMJ (Clinical Research Ed.) 2011, 342, d2807. https://doi.org/10.1136/bmj.d2807

Der Perre, L., Ness, R., Thoen, S., Vandenameele, P. y Engels, M. (2002). Understanding the indoor environment. En M. Engels (Ed.), Wireless OFDM Systems: How to make them work? (pp. 11-31). Boston, MA: Springer US. https://doi.org/10.1007/0306-47685-1_2

(EnAct). (n.d.). The ENERGY ACTION project. Recuperado de http://www.coldathome.today/.European Comission. (2014). European Union Statistics on Income and Living Conditions (EUSILC).

European Commission (n.d.). Energy Roadmap 2050. Communication from the Commission to the European Parliament, the Council, the European Economic And Social Committee and the Committee of the Regions, 1-20. https:// doi.org/10.2833/10759

European Commission. (2018). EU Energy Poverty Observatory (CN ENER/B3/SER/2015-507/SI2.742529). Recuperado de https://www.energypoverty.eu/

Fabbri, K. (2015). Building and fuel poverty, an index to measure fuel poverty: An Italian case study. Energy, 89, 244-258. https:// doi.org/10.1016/j.energy.2015.07.073
Florio, P. y Teissier, O. (2015). Estimation of the Energy Performance Certificate of a housing stock characterised via qualitative variables through a typology-based approach model: A fuel poverty evaluation tool. Energy and Buildings, 89, 39-48. https://doi.org/10.1016/j.enbuild.2014.12.024

Gatto, A. y Busato, F. (2020). Energy vulnerability around the world: The global energy vulnerability index (GEVI). Journal of Cleaner Production, 253, 118691. https://doi.org/https://doi. org/10.1016/j.jclepro.2019.118691

Gouveia, J. P., Palma, P. y Simoes, S. G. (2019). Energy poverty vulnerability index: A multidimensional tool to identify hotspots for local action. Energy Reports, 5, 187-201. https://doi.org/ https://doi.org/10.1016/j.egyr.2018.12.004

Hatt, T., Saelzer, G., Hempel, R. y Gerber, A. (2012). High indoor comfort and very low energy consumption through the implementation of the Passive House standard in Chile. Revista de La Construccion 2012, 11(2), 123-134. https://doi. org/10.4067/S0718-915X2012000200011

Heijs, W. y Stringer, P. (1988). Research on residential thermal comfort: some contributions from environmental psychology. Journal of Environmental Psychology, 8(3), 235-247. https://doi. org/10.1016/S0272-4944(88)80012-4

Heindl, P. (2015). Measuring Fuel Poverty: General Considerations and Application to German Household Data. Finanz Arch 2015, 71(2), 178-215. https://doi.org/10.1628/0015 $22115 \times 14285723527593$.

Herrero, S. T. (2017). Energy poverty indicators: A critical review of methods. Indoor and Built Environment, 26(7), 1018-1031. https://doi.org/10.1177/1420326X17718054

Hills, J. (2012). Getting the measure of fuel poverty. Final Report of the Fuel Poverty Review. Centre for the Analysis of Social Exclusion. London.

Howden-Chapman, P., Viggers, H., Chapman, R., O'Sullivan, K., Telfar Barnard, L., y Lloyd, B. (2012). Tackling cold housing and fuel poverty in New Zealand: A review of policies, research, and health impacts. Energy Policy 2012, 49, 134-142.

Isherwood, B.C. y Hancock, R. M. (1979). Household expenditure on fuel: distributional aspects. Economic Adviser's Office, DHSS, London.

Kolokotsa, D. y Santamouris, M. (2015). Review of the indoor environmental quality and energy consumption studies for low income households in Europe. Science of the Total Environment 2015, 536(February 2016), 316-330. https://doi.org/10.1016/j. scitotenv.2015.07.073

Legendre, B. y Ricci, O. (2014). Measuring fuel poverty in France: which households are the most vulnerable? Energy Economics 2014, 49, 620-628. https://doi.org/10.1016/j. eneco.2015.01.022

Liddell, C. y Morris, C. (2010). Fuel poverty and human health: A review of recent evidence. Energy Policy 2010, 38(6), 29872997. https://doi.org/10.1016/j.enpol.2010.01.037 
Longhurst, N. y Hargreaves, T. (2019). Emotions and fuel poverty: The lived experience of social housing tenants in the United Kingdom. Energy Research \& Social Science, 56, 101207. https:// doi.org/https://doi.org/10.1016/j.erss.2019.05.017

M. Bluyssen, P. (2014). The Healthy Indoor Environment: How to assess occupants' wellbeing in buildings. New York: Routledge.

Martínez, P. W. y Kelly, M. T. (2015). Integration of performance criteria in the energy-environmental improvement of existing social housing in Chile. Ambiente Construído 2015, 15(2), 47-63. https://doi.org/10.1590/s1678-86212015000200013

Meszerics, T. (ed.) (2016). Energy Poverty Handbook. Brussels: European Union.

Middlemiss, L. (2016). A critical analysis of the new politics of fuel poverty in England. Critical Social Policy, 37(3), 425-443.

Miniaci, R., Scarpa, C. y Valbonesi, P. (2014). Fuel Poverty and the Energy Benefits System: The Italian Case. (No. 66). Milano.

Moore, R. (2012). Definitions of fuel poverty: Implications for policy. Energy Policy 2012, 49, 19-26. https://doi.org/10.1016/j. enpol.2012.01.057

O'Brien, G. y Hope, A. (2010). Localism and energy: Negotiating approaches to embedding resilience in energy systems. Energy Policy, 38(12), 7550-7558. Recuperado de http://nrl.northumbria. ac.uk/8515/1/Localism_and_Energy_Negotiating_approaches_ to_embedding_resilience_in_energy_systems.pdf

Observatorio de Pobreza Energética en México. (n.d.). Retrieved June 15, 2020, from https://pobrezaenergetica.mx/inicio

Ortiz, J., Casquero-Modrego, N. y Salom, J. (2019). Health and related economic effects of residential energy retrofitting in Spain. Energy Policy, 130, 375-388. https://doi.org/https://doi. org/10.1016/j.enpol.2019.04.013

Paugan, S. (1995). The spiral of precariousness: a multidimensional approach to the process of social disqualification in France. Policy Press, (47996), 47-79.

Peneva, T. (2016). Mechanism for Protection of Vulnerable Consumers in Bulgaria. Recuperado de http://fuelpoverty. eu/2016/10/18/mechanism-for-protection-of-vulnerableconsumers-in-bulgaria/

Pérez-Fargallo, A., Rubio-Bellido, C., Pulido-Arcas, J. A. y Trebilcock, M. (2017). Development policy in social housing allocation: Fuel poverty potential risk index. Indoor and Built Environment, 26(7), 980-998. https://doi.org/10.1177/1420326X17713071

Porras-Salazar, J. A., Contreras-Espinoza, S., Cartes, I., PiggotNavarrete, J. y Pérez-Fargallo, A. (2020). Energy poverty analyzed considering the adaptive comfort of people living in social housing in the central-south of Chile. Energy and Buildings, https://doi. org/10.1016/j.enbuild.2020.110081

Rademaekers, K., Yearwood, J., Ferreira, A., Pye, S., lan Hamilton, P., Agnolucci, D. G., ... Anisimova, N. (2016). Selecting Indicators to Measure Energy Poverty. Rotterdam.
Red de Pobreza Energética (RedPE). Universidad de Chile. (2017). Recuperado de http://redesvid.uchile.cl/pobreza-energetica/

Romero, J. C., Linares, P., López Otero, X., Labandeira, X. y Pérez Alonso, A. (2015). Energy poverty in Spain. Economic analysis and proposals for action. Economics for Energy. Madrid.

Rosenow, J., Platt, R. y Flanagan, B. (2013). Fuel poverty and energy efficiency obligations - A critical assessment of the supplier obligation in the UK. Energy Policy 2013, 62, 1194-1203. https:// doi.org/10.1016/j.enpol.2013.07.103

Sánchez-Guevara Sánchez, C., Neila Gonzalez, F. J. y Hernández Aja, A. (2014). Towards a fuel poverty definition for Spain. In World Sustainable Building Conference (pp. 11-17). Barcelona: World Sustainable Building Conference 2014.

Sánchez-Guevara Sánchez, C., Neila González, F. J. y Hernández Aja, A. (2018). Energy poverty methodology based on minimal thermal habitability conditions for low income housing in Spain. Energy and Buildings, 169, 127-140. https://doi.org/https://doi. org/10.1016/j.enbuild.2018.03.038

Schuessler, R. (2014). Energy Poverty Indicators: Conceptual Issues. Centre for European Economic Research (ZEW), Discussion Paper Series 2014, 14(14), 37.

Shonali Pachauri, D. S. (2004). Energy Use and Energy Access in Relation to Poverty. Economic and Political Weekly 2004, 39(3), 271-278. Recuperado de http://www.jstor.org/stable/4414526

Sokołowski, J., Lewandowski, P., Kiełczewska, A. y Bouzarovski, S. (2020). A multidimensional index to measure energy poverty: the Polish case. Energy Sources, Part B: Economics, Planning, and Policy, 1-21. https://doi.org/10.1080/15567249.2020.1742817

Spanish National Statistics Institute. (2014). Survey on Living Conditions (SLC). Madrid.

Thomson, H., Snell, C. y Liddell, C. (2016). Fuel poverty in the European Union: a concept in need of definition? People, Place \& Policy, 10/1, 5-24. https://doi.org/10.3351/ppp.0010.0001.0002

Tirado Herrero, S., Jiménez Meneses, L., López Fernández, J. L., Martín Gracía, J. y Perero Van Hove, E. (2014). Energy poverty in Spain. Trend analysis. (Asociación de Ciencias Ambientales (ACA), Ed.) (1 ${ }^{\text {a.). Madrid. }}$

Tirado Herrero, S. y Ürge-Vorsatz, D. (2012). Trapped in the heat: A post-communist type of fuel poverty. Energy Policy, 49, 60-68. https://doi.org/10.1016/j.enpol.2011.08.067

Van Hooff, T., Blocken, B., Hensen, J. L. M. y Timmermans, H. J. P. (2015). Reprint of: On the predicted effectiveness of climate adaptation measures for residential buildings. Building and Environment 2015, 83, 142-158. https://doi.org/10.1016/j. buildenv.2014.10.006

Vilches, A., Barrios Padura, Á. y Molina Huelva, M. (2017). Retrofitting of homes for people in fuel poverty: Approach based on household thermal comfort. Energy Policy, 100, 283-291. https://doi.org/10.1016/j.enpol.2016.10.016 
Walker, R., Liddell, C., McKenzie, P. y Morris, C. (2013). Evaluating fuel poverty policy in Northern Ireland using a geographic approach. Energy Policy 2013, 63, 765-774. https://doi. org/10.1016/j.enpol.2013.08.047

Walker, R., McKenzie, P., Liddell, C. y Morris, C. (2012). Area-based targeting of fuel poverty in Northern Ireland: An evidenced-based approach. Applied Geography 2012, 34, 639-649. https://doi. org/http://dx.doi.org/10.1016/j.apgeog.2012.04.002

Walker, R., McKenzie, P., Liddell, C. y Morris, C. (2014). Estimating fuel poverty at household level: An integrated approach. Energy and Buildings 2014, 80, 469/479. https://doi.org/10.1016/j. enbuild.2014.06.004

Welsh, M. (2014). Resilience and responsibility: governing uncertainty in a complex world. The Geographical Journal, 180(1), 15-26. https://doi.org/10.1111/geoj.12012 